\title{
The social gradient in stress and depressive symptoms among adolescent girls: A systematic review and narrative synthesis
}

\author{
Janne Lund, Anders J.W. Andersen and Siri H. Haugland \\ Department of Psychosocial Health, University of Agder, Norway \\ Correspondence Janne Lund, Department of Psychosocial Health, Faculty of Health and Sport Sciences, University of Agder, Norway \\ Email: janne.lund@uia.no Telephone: +4799640729
}

\begin{abstract}
Aim: Socioeconomic inequality is found to negatively influence mental health, but studies investigating the relationship between socioeconomic status (SES) and specific common mental health problems such as stress and depressive symptoms in the general adolescent population are needed. Moreover, gender gaps in mental health among adolescents are evident, but there is a lack of studies that investigate socioeconomic differences in mental health within genders. As girls report consistently more depressive symptoms than do boys, this systematic review specifically investigates whether socioeconomic status is associated with stress and depressive symptoms among adolescent girls in the general population.

Methods: Eligible studies according to predefined inclusion criteria were identified from Medline, PsycINFO, ISI Web of Science, Svemed+ and Idunn. Eight studies were identified, whereby only two measured stress; hence, the evidence base for stress was too limited to perform an analysis. A narrative synthesis was conducted of the six studies that measured depressive symptoms.

Results: A significant inverse social gradient in depressive symptoms among adolescent girls was revealed in all studies that applied parental employment status and perceived financial difficulties as SES measures, while parental educational level and Family Affluence Scale (FAS) gave inconsistent results. The relatively low number of studies may limit interpretation.

Conclusions: Depressive symptoms were more common among adolescent girls with low SES compared to girls with higher SES. SES measures should be applied with care in studies of populations of adolescent girls, as the results can vary based on the chosen indicator. Actions to reduce depressive symptoms among adolescent girls in the general population should include targeting socioeconomic inequalities.
\end{abstract}

This is an open access article distributed under the Creative Commons Attribution Licence, which permits unrestricted use, distribution, and reproduction in any medium, provided the original work is properly cited.

\section{BACKGROUND}

It has long been claimed that socioeconomic status (SES) is a fundamental cause of disease (1), and the negative association between socioeconomic status and health problems is known as the social gradient in health, meaning that health problems increase as socioeconomic status decreases (2). Moreover, it has been established that greater social inequality within a society predicts more mental health problems for all, but more so for people with lower SES (3). Consequently, the social gradient in health is likely to be steeper in societies with higher social inequality.

The social gradient in health is evident in the context of adult mental health (3), and a systematic review (4) also showed an inverse relationship between socioeconomic background and general mental health problems among children and adolescents. However, previous research also reveals some inconsistency in the relationship between SES and adolescent mental health (4-6). It has been suggested that this variation in results may be caused by the application of different SES and mental health measures (4). Looking specifically at existing research on stress or depressive symptoms among adolescents, a social gradient is present in some studies (8-12) but not in others $(7,13)$. Although a social gradient in children and adolescent general mental health have been found, a systematic approach to investigate the significance of SES related to specific mental health problems is relevant (4).

According to recent research, internalizing problems among girls are increasing (14), and an increasing proportion of adolescent girls are reporting depressive symptoms (15-17). Moreover, girls report consistently more depressive symptoms than do boys $(17,18)$. The gender gap in the reporting of subjective health symptoms among adolescents is internationally recognized (19-21) and the evidence base for a gender gap in symptoms of stress is growing $(7,9,22)$. Such gender gaps demand research that explores gender perspectives more deeply $(23,24)$, especially with regard to socioeconomic status, as girls and boys might be differently affected by SES (25) and various SES dimensions (9). Gender-nuanced analysis is also required in order to avoid gender bias (23). The amount of gender-nuanced research on girls and the social gradient in stress or depressive symptoms is rather limited, and the topic has not been examined sufficiently. Two existing reviews $(4,11)$ did not find a significant gender difference in the social gradient; however, neither reviews examined the social gradient within the female population. Therefore, it is essential to expand the focus from only comparing and contrasting results on gender in order to explore variations within gender. 
Table 1. PICOC Schema, blocks and terms for systematic search.

\begin{tabular}{|c|c|c|}
\hline \multirow{5}{*}{$\begin{array}{l}\text { Population: } \\
\text { Adolescent girls (aged 13-18) }\end{array}$} & Keywords for all databases & Mesh terms for respective databases \\
\hline & Adolescen* OR & Human Female (PsycINFO) \\
\hline & Youth* OR & Adolescent (Medline, Svemed + ) \\
\hline & Teenager* AND & \\
\hline & $\begin{array}{l}\text { Girl* OR } \\
\text { Female* }\end{array}$ & \\
\hline \multirow{7}{*}{$\begin{array}{l}\text { Intervention/Comparison: } \\
\text { Socioeconomic status }\end{array}$} & Socioeconom* OR & Socioeconomic Status (P) \\
\hline & "Social Class*" OR & Social Class $(\mathrm{M} ; \mathrm{S})$ \\
\hline & Social inequalit* OR & Socioeconomic Factors (M; S) \\
\hline & "inequality*" NEAR3 (social.mp. or income.mp. or & \\
\hline & education*.mp.) OR & \\
\hline & Poverty OR & \\
\hline & "Educational status*" & \\
\hline \multirow{4}{*}{$\begin{array}{l}\text { Outcome: } \\
\text { stress and depressive } \\
\text { symptoms }\end{array}$} & Stress* OR & Distress (P, under STRESS) \\
\hline & Cortisol AND & Stress, psychological (M; P; S) \\
\hline & Depress* OR & Depressive, disorder (M) \\
\hline & Feeling low & $\begin{array}{l}\text { Depression }(\mathrm{M}) \\
\text { depressive; }(\mathrm{TS}=\mathrm{W})\end{array}$ \\
\hline $\begin{array}{l}\text { Context: } \\
\text { Geography }\end{array}$ & $\begin{array}{l}\text { Western countries (Europe, North America, Australic } \\
\text { search-filter. Filtering on geography was performed }\end{array}$ & $\begin{array}{l}\text { and New Zealand). No available } \\
\text { anually during the screening process. }\end{array}$ \\
\hline
\end{tabular}

Moreover, there is a need for specificity when discussing mental health issues and for nuance when discussing SES measures (4). Consequently, this current study treats socioeconomic status as individual SES factors and specifies mental health as either stress or depressive symptoms. Stress and depressive symptoms are closely linked, and stress is often the precursor to depression $(26,27)$. Moreover, this review focuses on a broad spectrum of symptom levels and on a non-clinical, general population of adolescent girls.

\section{Aim}

The aim of this systematic review is to investigate whether socioeconomic status is associated with stress and/or depressive symptoms in the general population of adolescent girls (age 13-18).

\section{METHODS}

This study investigates the relationship between stress and depressive symptoms and socioeconomic status (SES) among girls by performing a systematic review (28) (unregistered protocol) and narrative synthesis (29). The narrative synthesis is an appropriate tool to provide an overview of the presence of a possible social gradient in the included studies and to analyze the nuances between the respective studies (e.g. different cut-offs for depression, different contexts, different SES measures).

\section{Search strategy and study selection}

A systematic literature search was performed in March 2017 with no limit backwards in time. Articles were collected from electronic databases and through manual searches. The following databases were included in the search process: PsycINFO, Medline, ISI Web of
Science, Svemed+, and Idunn. MesH terms and keywords were included in the systematic search. The explode function was used on Mesh terms. In addition, the focus function was used on MesH terms for SES, depression and stress. The strategy of the search is displayed in Table 1. The search string applied in Medline was as follows:

exp Adolescent/ OR adolescen*.mp. OR Youth*.mp. OR teenager*.mp. AND (girl* or female*).mp. AND exp *Social Class/ or exp *Socioeconomic Factors/ or socioeconom*.mp. or "social class*".mp. OR (inequalit* adj3 (social* OR income* OR education*)).mp. OR "educational status*".mp. OR exp *Poverty/ OR poverty.mp. AND exp. *Stress, Psychological/ OR stress*.mp. OR cortisol*.mp. OR exp *Depression/ or depress*.mp. OR exp *Depressive Disorder/ OR *feeling low*.mp. OR (low adj3 mood).mp. Limit to (Danish or English or Norwegian or Swedish) and "adolescent (13 to 18 years)"

Regarding the manual search, the reference lists of included articles were screened, as well as the publication lists of included first-authors, diverse Scandinavian journals, and web pages of institutions. Experts in the field were also contacted (Norwegians) with request for additional research.

\section{Inclusion of studies}

The inclusion criteria were set to identify peer reviewed articles that provided information on the relationship between SES and stress and/or depressive symptoms among adolescent girls. SES must be applied at the individual level, and articles focusing only on area/neighborhood SES were excluded. For quantitative studies, SES must be applied as an explanatory variable. Moreover, stress and/or depressive symptoms should be a central topic and must be measured as the 


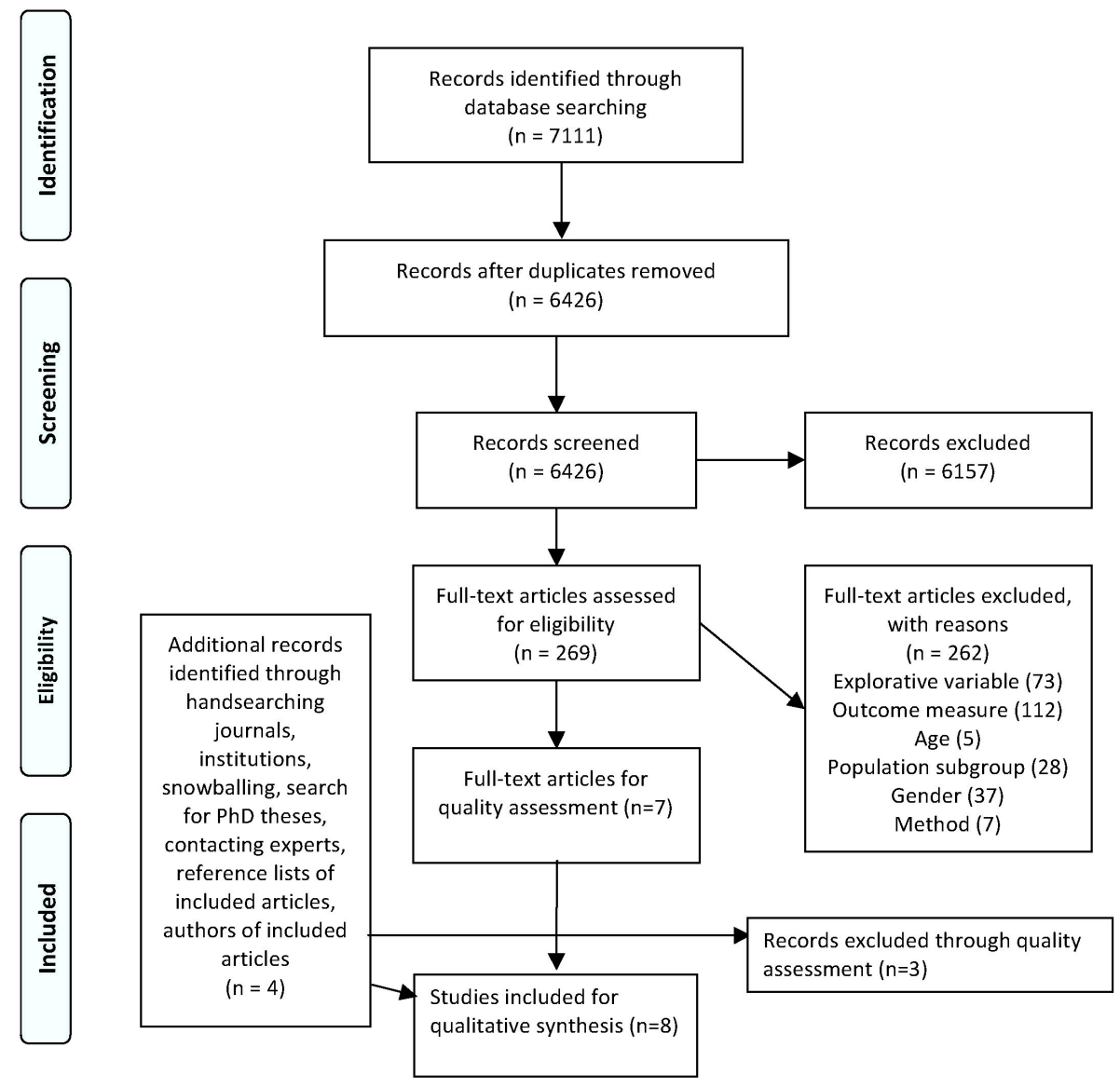

Figure 1. PRISMA flow diagram.

outcome for quantitative studies. Articles dealing only with clinical depression or other mental issues were not included, nor were studies focusing solely on diagnostic measuring scales. Moreover, the sample in the study must be adolescents, 13-18 years old, from the general population, and studies focusing solely on population subgroups were excluded. The included studies must also provide gender-nuanced results by providing specific information about the associations between SES and stress and/or depressive symptoms within the female population.

A total of 7111 articles were identified, and 6426 remained after removal of duplicates. According to predefined inclusion and exclusion criteria, 6157 articles were excluded after screening titles and abstracts. This step was carried out by one researcher. Thereby, 269 articles were full-text screened by two researchers. The screening was performed in the software Covidence (30). Seven articles met the inclusion criteria, and four additional records were identified from the manual search: three reports and one article. Critical appraisal lists from the Joanna Briggs Institute (31) were used by two researchers to assess the quality of all included studies. The three reports were excluded because they did not maintain the proper analytical standard. Finally, eight research articles were included for extraction and analysis. The PRISMA flow diagram in Figure 1 illustrates this process (32).

\section{Data extraction}

The following information was extracted from each included article: Authors, title, year of publication, year of data collection for outcome, country of data collection, study and analytical design, name of study, population age group, number of respondents (girls), measure of socioeconomic status, measure of outcome, main findings in text and in numbers concerning the association between SES and outcome in girls, and summary of findings of a social gradient. Two of the included studies were cross-sectional trend studies with several measure points $(16,17)$, from which the newest available data were extracted for analysis.

Table 2 provides an overview of the included articles. Only two measured perceived stress as an outcome; therefore, the following synthesis is based on the six studies of depressive symptoms. The problem of the inadequate evidence base concerning girls, SES and perceived stress is examined in the discussion chapter.

\section{Analysis: Narrative synthesis}

To create an initial overview of the main results, the studies and their respective conclusions were grouped into two main categories: 1) no findings of a significant social gradient and 2) findings of a significant social gradient. When studies included more than one SES measure the results were split into several lines to see the separate effects of each measure. The choice to 
Table 2. Overview of included studies alphabetically listed by author.

Title, country and study design

Bulhões et al. 2013. Depressive symptoms and its associated factors in 13-year-old urban adolescents. Portugal.

Design: Cross sectional, sample size (girls) 1037; Socioeconomic status (SES) measure: Parental education; Outcome: Depressive symptoms, Beck Depression Inventory (BDI) (33).

Dahlqvist et al. 2012. Depressive symptoms and the associations with individual, psychosocial, and structural determinants in Swedish adolescents. Sweden.

Design: Cross sectional, sample size 628; SES: Family Affluence Scale (FAS), "Money as friends"; Outcome: Depressive symptoms, Center for Epidemiologic Studies Depression Scale (CES-D) (34).

Frojd et al. 2006. Perceived financial difficulties and maladjustment outcomes in adolescence. Finland.

Design: Cross sectional, sample size 1609; SES: Perceived financial difficulties, Parents' education, Parents' employment status; Outcome: Depressive symptoms, BDI (5).

Gariepy and Elgar 2016. Trends in Psychological Symptoms among Canadian Adolescents from 2002 to 2014: Gender and Socioeconomic Differences. Canada.

Design: Cross sectional, Trend, sample size 13813; SES: FAS; Outcome: Depressive symptoms, Health Behavior in School-aged children checklist (HBSC), item "feeling low or depressed" (16).

Glasscock et al. 2013. Can negative life events and coping style help explain socioeconomic differences in perceived stress among adolescents? A cross-sectional study based on the West Jutland cohort study. Denmark.

Design: Cross sectional, sample size 1456; SES: Parental education and income; Outcome: Stress, Cohen's Perceived Stress Scale (PSS) (9).

Gore et al. 1992. Social Structure, Life Stress, and Depressive Symptoms in a High SchoolAge Population. US.

Design: Cross sectional, sample size 675; SES: Parent education attainment, Perceived standard of living; Outcome: Depressive symptoms, CES-D (35).

Landstedt and Gadin 2012. Seventeen and Stressed - Do gender and class matter? Sweden. Design: Cross sectional, sample size 815; SES: Academic Orientation in adolescents; Outcome: Stress, Perceived Stress "How often during the past 3 months have you felt stressed?" (7).

Torikka et al. 2014. Self-reported depression is increasing among socio-economically disadvantaged adolescents - repeated cross-sectional surveys from Finland from 2000 to 2011. Finland.

Design: Cross sectional, Trend, sample size 51006; SES: Parental education and employment status; Outcome: Depressive symptoms, BDI (17).
Main findings in female population

No significant correlation between education and depressive symptoms in girls in adjusted models, only in crude model.

No significant association between depressive symptoms in girls and FAS.

"Money as friends?" was independently associated with depressive symptoms and shows a social gradient.

Significant association and negative social gradient in both perceived financial difficulties and parents' employment status and depressive symptoms.

Significant association and a negative social gradient in FAS and "feeling low or depressed" between the low and medium/high FAS group.

Significant social gradient in household income and perceived stress among girls. Significant social gradient in parental education and perceived stress.

Significant social gradient in parent education attainment and Perceived Standard of Living.

No significant association, and no significant social gradient, between Academic Orientation and perceived stress.

Significant social gradient in employment status and educational level combined. The relative odds of severe depression were higher for those with low-educated and unemployed parents than for those with high-educated and employed parents. split the studies in this way was founded on Reiss' (4) statement that there are great variations in outcomes based on the different SES measures. Out of the six studies in our synthesis, ten results on the association between SES and depressive symptoms were now displayed as shown in Table 3 . Seven results revealed a significant association, while three did not.

To identify similarities or contradictions between the ten results in relation to the presence of a significant gradient or no findings of a significant gradient, more details were added in the overview schema. The additional details were the outcome measure scale, the analytical measure (e.g. odds ratio), whether the SES information were reported by adolescents or parents, the age of adolescents and the country of the study.
After applying the detailed study characteristics, the ten results were systematized by different strategies. The first systematization, by SES measure, is displayed in Table 4. The different SES measures from the included studies were grouped into five categories: 1) Parental education level (including maternal and paternal education, parent with the highest education); 2) Family affluence scale (FAS); 3) Employment status (unemployment of one or both parents); 4) Perceived financial difficulties (including perceived financial difficulties, perceived standard of living and the item "Do you have the same money as your friends?") and 5) Parental education and employment status combined. After categorizing by SES measure, the results were systematized in a similar manner by 


\section{Results by socioeconomic status}

There was a pattern regarding what type of SES measure was applied in the studies and whether the results of the respective studies showed a significant social gradient.

Three studies measured educational level in parents as a separate measure $(5,33,35)$, whereby only one of them (35) found a significant association between parental education and depressive symptoms among girls. Two studies measured Family Affluence Scale (FAS) $(16,34)$, whereby only one found a significant association between FAS and depressive symptoms (16). One study measured employment status as a separate measure and found a significant social gradient (5). The measure of perceived financial difficulties was applied in three studies $(5,34,35)$, and a significant social gradient was revealed in all studies. One study (17) measured parental education level and parental employment status in combination and found a significant social gradient. In the latter mentioned study, the gradient remained when girls with employed, highly educated parents were compared to girls with employed, low-educated parents, thus indicating a separate gradient for parental education. Moreover, the gradient remained when girls with employed, highly educated parents were compared to girls with unemployed, highly educated parents, thus indicating a separate gradient for parental employment status. In general, for all studies, whether the SES measure was dichotomized or trichotomized did not seem to affect the gradient systematically.

To summarize, the social gradient might depend on which measure of SES is used in the study. The measure of parental employment status and perceived financial difficulties gave consistent results and revealed a social gradient. The SES measure of parental education level and FAS revealed non-consistent results.

\section{Comparisons of studies with parental educational level or FAS as a SES measure}

The comparison is based on the above mentioned detailed study characteristics and analytical details. However, in the following section, only those aspects are referred to that represented differences between studies with a significant gradient and studies with no findings of a significant gradient.

Four studies included parental education as a SES measure; Bulhões et al. (33) and Frojd et al. (5) did not find a significant social gradient, while Gore et al. (35) and Torikka et al. (17) found a significant social gradient. Gore et al. (35) used the Center for Epidemiologic Studies' Depression Scale (CES-D), with a cut-off score of 24 that indicated clinical depression for adolescent girls. CES-D is a depression scale for research in the general population (36). The three other studies used the Beck Depression Inventory (BDI) as an outcome measure, which is a self-reporting tool that measures attitudes and symptoms of depression (37). However, the three studies were operating with diffe- rent cut-offs. Bulhões et al. (33) and Frojd et al. (5), who did not find a significant gradient, used cut-offs that merged, respectively, mild, moderate and severe symptoms and moderate and severe symptoms. Whilst Torikka et al. (17), who found a significant gradient between low and high education, operated with three different cut-offs and provided a separate analysis for each level (mild, moderate, severe) of symptoms. That study also had a radically higher number of respondents $(\mathrm{n}=51006)$ than both Bulhões et al. $(33)(\mathrm{n}=1037)$ and Frojd et al. (5) (n=1609). Gore et al. (35) had 675 participants. Additionally, Torikka et al. (17), who found a social gradient, measured the educational level of parents in combination with employment status and it was not possible to split the two factors. However, the gradient seemed to be present for parental educational level separately as well as in the merged measure. It is also worth mentioning that Bulhões et al. (33) actually did find a significant social gradient in the crude model; however, when the model was adjusted it was no longer significant.

Two studies used the FAS as the measurement tool, whereby the study from Dahlqvist et al. (34) revealed no significant association between FAS and depressive symptoms among adolescent girls and the study from Gareipy and Elgar (16) did reveal a significant gradient between low and high FAS. There were several differences between the two studies. First, they used different measuring scales for depressive symptoms. Dalqvist et al. (34) used the Center for Epidemiologic Studies' Depression Scale (CES-D) with a cut-off score that indicated clinical depression (38). Gariepy and Elgar (16), who found a social gradient, were using the Health Behavior in School-aged Children checklist (HBSC), which is a checklist that measures a broad range of aspects of the lives of children and adolescents, including internalizing symptoms (39). They used a cut-off that indicated symptoms occurred weekly or more often and they split the results into specific symptoms, whereby only the item "feeling low or depressed" was extracted for the synthesis in this review. Second, Dahlqvist et al. (34), who did not find a significant social gradient, had a narrower age group (age 13-16 versus age 10-19), third, a lower number of respondents $(\mathrm{n}=628$ versus $\mathrm{n}=30117$ ), fourth, a different analytical measure (odds ratio versus probability score) and fifth, a different context (Nordic versus non-Nordic).

In sum, the comparison shows that within the SES group of educational level of parents, there are differences between the studies, but hardly any consistent pattern. Three studies used the same outcome score, BDI, whereby only one of them (17) found a significant social gradient. This study had a more nuanced outcome measure, with several cut-offs compared to the two other studies. It is also important to emphasize that this study measured educational status in combination with employment status, which of course makes this comparison difficult. Regarding the SES group of 
FAS, the study that found a social gradient had a significantly higher number of participants than did the other study.

\section{Strength of the gradient}

The significant gradients that were found regarding employment status and perceived financial difficulties were all quite strong $(5,34,35)$. The odds ratio $(\mathrm{OR})$ for depressive symptoms among girls with low SES was much higher than that among girls with high SES; specifically, it was approximately two times higher in all studies (high SES as reference group, OR 1, low SES ranging from OR $1.9 \mathrm{CI}$ [1.35-2.67] till $2.4 \mathrm{CI}$ [1.70-3.39]). One study applied multivariate linear regression, and found a gradient in depressive symptoms in relation to SES with an effect size of $b=-.200$ $(\mathrm{se}=.043, \mathrm{p} \leq .05)(35)$. It is worth mentioning that this effect was largely accounted for by exposure to life stress. A significant gradient was also found in FAS, where the predicted probability score increased from $0.20 \mathrm{CI}[0.18,0.21]$ in low FAS to $0.25 \mathrm{CI}[0.23,0.27]$ in high FAS (16).

The gradient that was found regarding parental educational level was significant, but however weaker than the gradient of perceived financial difficulties (perceived standard of living) found in the same study $(b=-.043$ versus $b=-.200)$ (35). When combining educational level with employment status, a profound gradient, OR 4.86, was present in severe depression when comparing low educated and unemployed parents with highly educated and employed parents. The gradient was significantly present in the two lower levels of depression as well, but with lower OR: 2.64 CI [2.32$3.00]$ in moderate and $1.93 \mathrm{CI}$ [1.69-2.20] in mild depression (17).

\section{DisCUSSION}

This systematic review investigated the social gradient in stress and depressive symptoms among adolescent girls. Eight studies were identified, but only two studies investigated the relationship between SES and stress. A narrative synthesis was therefore only conducted of the six studies that measured depressive symptoms. The majority of the included studies showed that depressive symptoms were more prevalent among girls with lower socioeconomic status compared to girls with a higher socioeconomic background. Findings were in line with previous research $(4,11)$ focusing on the social gradient related to adolescent mental health in general. However, some inconsistent relationships were found, and the narrative analysis suggests that this may be related to the different SES measures applied in the studies.

\section{SES measures and the partly inconsistent gradient in depressive symptoms}

The partly inconsistent relationship between various SES measures and depressive symptoms have already been noted by Reiss (4). In this current study, the outcome measures applied were relatively homogenous and were defined as depressive symptoms. However, different SES measures still provided different results and thereby support the suggestion regarding the importance of the SES measure itself. Even within the same studies, different SES measures provided opposite findings of a social gradient. For example, in Frojd et al. (5), the SES measure of adolescents' perceived financial difficulties provided a social gradient in depressive symptoms, while parental education did not provide a social gradient. The most consistent association was found in the SES measure of perceived financial difficulties and parental employment status. These measures may be considered above parental education when it comes to SES and female depressive symptoms.

The inconsistency in social gradient based on different SES measures corresponds with existing findings on the validity of SES measures. Svedberg et al. (6) recently found that the congruence between the measures of parent occupations, FAS and perceived SES among adolescents is low. This might indicate that SES should be viewed as a range of different dimensions possibly affecting adolescents in diverse ways. Moreover, when examining the association between the different SES measures and Health Related Quality of Life (HRQOL), a significant gradient was only found concerning perceived SES. Perceived SES is suggested to represent a wider construct of social status than the more traditional SES measures, hence it may affect adolescents more strongly (6). This is partly consistent with the findings from the synthesis on depressive symptoms in adolescent girls presented in this review, where the SES measure of perceived financial difficulties produced a social gradient. However, perceived financial difficulties is a narrower measure than general perceived socioeconomic status, which, again, might make this comparison inappropriate. Svedberg et al. did not find a significant gradient regarding parental occupations (6); however, through this systematic review, we found indications of such a gradient $(5,17)$. The family affluence scale, which according to this review revealed inconsistent results, has been the object of critical discussions concerning its validity as a measure of adolescents' SES (40). FAS is a scale that quickly becomes outdated; however, the newest version, FAS III, is currently validated in countries of various income levels (41). It was not clear which version of FAS was applied in the studies included in this review $(16,34)$. Moreover, it is essential to note that parental education level also gave inconsistent results. Why parental education is more relevant in some studies, but not in others, is not clear. For instance, education may in some areas have less relative importance if the general level of higher education level is low. Employment may in such cases make a better SES measure. In some cultures, males are the "breadwinners" within the family economy, leaving 
low maternal education to be less significant in the relative socioeconomic status in the local community. Although all studies included are from western society, inequality between genders still vary among countries (42). Parents' education also seemed to be less important than being employed or unemployed regarding the social gradient in depressive symptoms among adolescent girls. This finding contrasts with Reiss' (4) review, where parental education had a stronger impact than employment status.

The inconsistency related to the associations between SES and depressive symptoms may reflect a situation where these associations actually differ. However, in the analysis presented in this systematic review we have looked for patterns within and between the included studies in order to shed light over the findings. It was difficult to find a consistent pattern that explained the differences in outcomes more convincingly than does the explanation of differentiation in SES measures. Concerning the analysis of comparisons within the SES groupings with inconsistent results, it was also difficult to find an internal pattern in the differences. The number of participants probably may have affected the results along with different outcome measures and differences in cut-off scores for the outcome measures. However, a consistent pattern was not revealed.

The inconsistency of different SES measures has implications for the use of SES indicators in research with populations of adolescent girls. One cannot manage SES as a homogenous measure because the specific SES indicator will affect the results. If controlling for SES by applying, for instance, parental education, the controlling might not be representative for other aspects of SES. If conducting reviews or synthesis of existing research, the same problem is present; one must be specific about which SES indicator is applied, and it makes little sense to compare studies that use different measures of SES. Parental education as a measure of SES should be further examined and applied with care. As shown in this review, this measure gives inconsistent results.

\section{Stress and socioeconomic status}

Only two articles about stress met the inclusion criteria, which was not sufficient to conduct an analysis. However, the overall pattern of different SES measures causing different outcomes was present in these two studies, as they operated with different SES measures and one study found a significant social gradient whilst the other one did not. The inconsistency in results can also be observed in studies that are not gender nuanced. For example, one study found a social gradient concerning stress among adolescents in general when using parents' educational level as a SES measure (8). Another study that measured parental education, income and perceived socioeconomic status revealed some indications of a social gradient; however, there were variations between SES measures and variations concerning race or ethnicity (10). When measuring cortisol and not perceived stress, the social gradient was not present (43).

One explanation for the insufficient amount of research regarding socioeconomic status and stress among adolescent girls is that stress research is often focused on stressors $(7,44)$ and on major stress events (45). Perhaps it is time to focus research on the general feeling of perceived stress among girls. Moreover, the critique has been made that there is a lack of measurement scales concerning adolescents and stress. The Adolescent Stress Scale (ASQ) was developed as a response to these critiques (26); however, it was found to require further examination in order to meet a sufficient standard (22).

\section{Social inequality and social gradient}

It seems conspicuous that no recent studies from highinequality countries were included in this review. The context for the included studies was limited to the western world: Europe, North America, Australia and New Zealand. This geographical definition included the US, which is a country of high inequality, as well as several countries from Europe with different levels of inequality (3). However, only two studies from high-inequality countries met the criteria, one study from Portugal and one quite old study from the US, and consequently the included studies were mainly from countries with low inequality. The inclusion criterion that studies should not have a sub-group population excluded quite a few studies from the US, where the sample is often an ethnic or socioeconomic subgroup. According to theories on social inequality, mental health problems should be more present in countries with high inequality, and the gradient would also possibly be more urgent because, while inequality increases mental health problems for all, people of the lowest SES are most affected (3). From the studies included in this review, it is particularly interesting to note that there was still a social gradient in depressive symptoms among adolescent girls, even when the studies represented so-called low-inequality countries. Additionally, Torikka et al. (17) found that depressive symptoms among girls were increasing more in socially disadvantaged groups than in other groups and suggested that this unequal increase is caused by increasing economic inequalities. Consequently, actions to decrease depressive symptoms among adolescent girls could be successfully implemented at a socioeconomic level.

\section{Strengths and weaknesses of the review}

A major strength of this study is the synthesis of gender-nuanced knowledge on the relationship between SES and depressive symptoms within the population of adolescent girls. The study also provides an overview of the effect of the different SES measures and reveals the importance of the choice of SES indicator. Moreover, the study reveals a lack of knowledge 
regarding SES and stress among adolescent girls.

Few studies were included in the final analysis, and the basis for drawing solid conclusions is probably too limited. There were several reasons why so few studies met the inclusion criteria. In general, there is a lack of studies investigating health differences within genders (46). Often studies make comparisons between males and females, or simply adjust for gender. This provides less gender-nuanced findings and is a challenge for health research within many fields and for the development of gender-nuanced interventions $(46,47)$. Further, many studies located in our initial search addressed limited populations (for example comparing ethnical groups), risk-groups or clinical samples. Many studies applied merged measuring scales and did not provide a symptom specific analysis, e.g. merging anxiety and depressive symptoms. Moreover, many studies applied clinical measures/diagnostic tools in order to define depression. As the prevalence of internalizing symptoms in the general population of adolescent girls seems to be increasing (14), the scope of the study was concerned around investigating SES in relation to these more common reports of mental health problems. Including clinical samples or studies applying diagnostic tools would probable increase the number of studies but reduce the possibility to extract knowledge about more general problems in a general population of adolescent girls. It would simply answer another research question than what was intended. The review also shows that gender-nuanced studies of a social gradient in common mental health problems in the general population is lacking, especially when it comes to stress. This is an interesting finding of its own and signifies the need for further research on these matters. The search could also be widened by including more languages; however, doing this would create some practical challenges. Additionally, in the context of western countries, one should assume that there would be enough research written in English, at least when including the US. However, the problem of the race/ ethnicity focus in the US, which leads to sub-group research, was more prominent than expected.
A general weakness with reviews of this type is that it is difficult to compare different studies because they contain too many differences and variations. Nevertheless, in this review, it is interesting to note that the differences in SES measures seem to overcome all the other contradictions between the studies. Concerning the results of the included studies, it might be a weakness that SES is mainly measured based on the statements of adolescents. It would be interesting to see more SES measures stated by parents and to include a measure of income. Moreover, all the included studies were cross-sectional studies, which implies that although there is a social gradient and an association between SES and depressive symptoms, one cannot - given the cross-sectional design - state anything about the causality of this association. Moreover, the lack of qualitative research on this topic is striking, probably because of the research question, where we addressed the relationship or association between two factors. However, it should be possible to investigate such a relationship using more qualitative in-depth methods.

\section{Conclusions}

Depressive symptoms were more common among adolescent girls with low socioeconomic status (SES) than higher socioeconomic status. However, findings varied with the different SES measures applied. There was a gradient in parental employment status and perceived financial difficulties. The SES measures of Family Affluence Scale (FAS) and parental educational level yielded inconsistent results and should be applied with care in studies of populations of adolescent girls. There is a striking need for gender-nuanced research on the association between SES and stress among the general population of adolescent girls. Moreover, actions to reduce depressive symptoms among adolescent girls in the general population should include targeting socioeconomic inequalities. This demands a nuanced understanding of the socioeconomic landscape.

\section{REFERENCES}

1. Link BG, Phelan J. Social conditions as fundamental causes of disease. J Health Soc Behav 1995;35:80-94.

2. Elstad JI. Den grunnleggende årsaken til sosial ulikhet i helse. In: Tjora A, editor. Helsesosiologi, 1. edn. Oslo: Gyldendal Akademisk, 2012, p. 350-70.

3. Wilkinson R, Pickett K. The spirit level: why equality is better for everyone. London: Penguin Books, 2010.

4. Reiss F. Socioeconomic inequalities and mental health problems in children and adolescents: A systematic review. Soc Sci Med 2013;90:24-31.

5. Frojd S, Marttunen M, Pelkonen M, von der Pahlen B, Kaltiala-Heino R. Perceived financial difficulties and maladjustment outcomes in adolescence. Eur J Public Health 2006;16(5):542-8.

6. Svedberg P, Nygren JM, Staland-Nyman C, Nyholm M. The validity of socioeconomic status measures among adolescents based on self-reported information about parents occupations, FAS and perceived SES; implication for health related quality of life studies. BMC Med Res Methodol 2016;16:48. 
7. Landstedt E, Gådin KG. Seventeen and stressed - Do gender and class matter? Health Sociol Rev 2012; 21(1):82-98.

8. Finkelstein DM, Kubzansky LD, Capitman J, Goodman E. Socioeconomic differences in adolescent stress: The role of psychological resources. J Adolesc Health 2007;40(2):127-34.

9. Glasscock DJ, Andersen JH, Labriola M, Rasmussen K, Hansen CD. Can negative life events and coping style help explain socioeconomic differences in perceived stress among adolescents? A cross-sectional study based on the West Jutland cohort study. BMC Public Health 2013;13:532.

10. Goodman E, McEwen BS, Dolan LM, Schafer-Kalkhoff T, Adler NE. Social disadvantage and adolescent stress. J Adolesc Health 2005;37(6):484-92.

11. Lemstra M, Neudorf C, D'Arcy C, Kunst A, Warren LM, Bennett NR. A systematic review of depressed mood and anxiety by SES in youth aged 10-15 years. Can J Public Health 2008;99(2):125-9.

12. Moor I, Richter M, Ravens-Sieberer U, Ottova-Jordan V, Elgar FJ, Pfortner TK. Trends in social inequalities in adolescent health complaints from 1994 to 2010 in Europe, North America and Israel: The HBSC study. Eur J Public Health 2015;25:57-60.

13. Twenge JM, Nolen-Hoeksema S. Age, gender, race, socioeconomic status, and birth cohort differences on the Children's Depression Inventory: A meta-analysis. J Abnorm Psychol 2002;111(4):578-88.

14. Bor W, Dean AJ, Najman J, Hayatbakhsh R. Are child and adolescent mental health problems increasing in the 21 st century? A systematic review. Aust N Z J Psychiatry 2014;48(7):606-16.

15. Collishaw S. Annual Research Review: Secular trends in child and adolescent mental health. J Child Psychol Psychiatry 2015;56(3):370-93.

16. Gariepy G, Elgar FJ. Trends in psychological symptoms among Canadian adolescents from 2002 to 2014 : Gender and socioeconomic differences. Can J Psychiatry 2016;61(12):797-802.

17. Torikka A, Kaltiala-Heino R, Rimpela A, Marttunen M, Luukkaala T, Rimpela M. Self-reported depression is increasing among socio-economically disadvantaged adolescents - repeated cross-sectional surveys from Finland from 2000 to 2011. BMC Public Health 2014;14:408.

18. von Soest T, Wichstrom L. Secular trends in depressive symptoms among Norwegian adolescents from 1992 to 2010. J Abnorm Child Psychol 2014;42(3):403-15.

19. European Comission. Report on the state of young peoples' health in the European Union. EU, 2000.

20. Hagquist C. Psychosomatic health problems among adolescents in Sweden - are the time trends gender related? Eur J Public Health 2009;19(3):331-6.

21. Ravens-Sieberer U, Torsheim T, Hetland J, Vollebergh W, Cavallo F, Jericek H, et al. Subjective health, symptom load and quality of life of children and adolescents in Europe. Int J Public Health 2009;54:151-9.

22. De Vriendt T, Clays E, Moreno LA, Bergman P, Vicente-Rodriguez G, Nagy E, et al. Reliability and validity of the Adolescent Stress Questionnaire in a sample of European adolescents - the HELENA study. BMC Public Health 2011;11:717.

23. Howard LM, Ehrlich AM, Gamlen F, Oram S. Gender-neutral mental health research is sex and gender biased. Lancet Psychiatry 2016;4(1):9-11.

24. Wiklund M. Close to the edge: discursive, gendered and embodied stress in modern youth [PhD Thesis]. Sweden: Umeå University, 2010.

25. Vikram P. Gender in Mental Health Research. Report. World Health Organization, 2004 January.

26. Byrne DG, Davenport SC, Mazanov J. Profiles of adolescent stress: The development of the adolescent stress questionnaire (ASQ). J Adolesc 2007;30(3):393-416.

27. Moksnes UK, Moljord IEO, Espnes GA, Byrne DG. The association between stress and emotional states in adolescents: The role of gender and self-esteem. Pers Individ Dif 2010;49(5):430-5.

28. Booth A, Sutton A, Papaioannou D. Systematic approaches to a successful literature review, 2nd edn. London: Sage; 2016.

29. Popay J, Roberts H, Sowden A, Petticrew M, Arai L, Rodgers M, et al. Guidance on the conduct of narrative synthesis in systematic reviews: A product from the ESRC Methods Programme. Lancaster: Lancaster University, 2006.

30. Cochrane. Covidence 2018 [cited 201704 April]. Available from: https://www.covidence.org/home.

31. JBI. Critical Appraisal Tools 2018 [cited 201811 October]. Available from: http://joannabriggs.org/research/critical-appraisal-tools.html.

32. Moher D, Liberati A, Tetzlaff J, Altman DG, PRISMA Group. Preferred reporting items for systematic reviews and meta-analyses: The PRISMA Statement. PLoS Med 2009;6(7).

33. Bulhoes C, Ramos E, Lindert J, Dias S, Barros H. Depressive symptoms and its associated factors in 13-yearold urban adolescents. Int J Environ Res Public Health 2013;10(10):5026-38.

34. Dahlqvist HZ, Landstedt E, Gådin KG. Depressive symptoms and the associations with individual, psychosocial, and structural determinants in Swedish adolescents. Health 2012;4(10):881-9. 
35. Gore S, Aseltine RH, Colton ME. Social-structure, life stress and depressive symptoms in a high school-aged population. J Health Soc Behav 1992;33(2):97-113.

36. Radloff LS. The CES-D Scale: A self-report depression scale for research in the general population. Appl Psychol Meas 1977;1(3):385-401.

37. American Psychological Association. Beck Depression Inventory 2018 [Available from: http://www.apa.org/pi/about/publications/caregivers/practice-settings/assessment/tools/beck-depression.aspx.

38. American Psychological Association. Center for Epidemiological Studies-Depression Scale 2018 [Available from: http://www.apa.org/pi/about/publications/caregivers/practice-settings/assessment/tools/depressionscale.aspx.

39. HBSC. Health Behaviour in School-aged Children: Survey Methods 2014 [Available from: http://www.hbsc.org/methods/index.html.

40. Boyce W, Torsheim T, Currie C, Zambon A. The family affluence scale as a measure of national wealth: Validation of an adolescent self-report measure. Soc Indic Res 2006;78(3):473-87.

41. Hartley JEK, Levin K, Currie C. A new version of the HBSC Family Affluence Scale - FAS III: Scottish Qualitative Findings from the International FAS Development Study. Child Indic Res 2016;9(1):233-45.

42. Forum WE. The Global Gender Gap Report 2018. Geneva: World Economic Forum, 2018. Contract No.: ISBN-13: 978-2-940631-00-1.

43. West P, Sweeting H, Young R, Kelly S. The relative importance of family socioeconomic status and schoolbased peer hierarchies for morning cortisol in youth: An exporatory study. Soc Sci Med 2010;70(8):1246-53.

44. Flouri E, Buchanan A, Tan JP, Griggs J, Attar-Schwartz S. Adverse life events, area socio-economic disadvantage, and adolescent psychopathology: The role of closeness to grandparents in moderating the effect of contextual stress. Stress 2010;13(5):402-12.

45. Eriksen IM, Sletten MA, Von Soest T. Stress og press blant ungdom. Oslo: NOVA/HIOA: Velferdsforskningsinstituttet, 2017 June.

46. Nowatzki N, Grant KR. Sex is not enough: The need for gender-based analysis in health research. Health Care Women Int 2011;32(4):263-77.

47. Tannenbaum C, Clow B, Haworth-Brockman M, Voss P. Sex and gender considerations in Canadian clinical practice guidelines: a systematic review. CMAJ Open 2017;5(1):E66-E73. 